\title{
Rapport du seminaire national [au Mali] pour la definition d'une strategie d'eradication de l'excision
}

Population Council

Follow this and additional works at: https://knowledgecommons.popcouncil.org/departments_sbsr-rh

Part of the Demography, Population, and Ecology Commons, Family, Life Course, and Society Commons, Gender and Sexuality Commons, International Public Health Commons, Obstetrics and Gynecology Commons, Public Health Education and Promotion Commons, and the Women's Health Commons

How does access to this work benefit you? Let us know!

\section{Recommended Citation}

"Rapport du seminaire national [au Mali] pour la definition d'une strategie d'eradication de l'excision," Rapport du Projet de Recherche Operationnelle et d'Assistance Technique en Afrique II. Bamako:

Population Council et République du Mali, Commissariat à la Promotion des Femmes, 1998. 
PRIMATURE

COMMISSARIAT A LA PROMOTION

DES FEMMES
REPUBLIQUE DU MALI UN PEUPLE UN BUT UNE FOI

\section{RAPPORT DU SEMINAIRE NATIONAL POUR LA DEFINITION D'UNE STRATEGIE D'ERADICATION DE L'EXCISION}

Commissariat à la Promotion des Femmes

Population Council

17, 18, 19 Juin 1997

Bamako, Mali

POPULATION COUNCIL RO/AT LOGO HERE

PROJET DE RECHERCHE OPERATIONNELLE ET

D'ASSISTANCE TECHNIQUE EN AFRIQUE II

JUIN 1998 


\section{Projet de Recherche Opérationnelle et d'Assistance Technique en Afrique II}

Les objectifs du Project de RO/AT Afrique II sont:

- élargir l'accès à une gamme étendue de services et méthodes de planification familiale

- tester différentes méthodes afin d'intégrer les services de santé en matière de reproduction aux programmes de planification familiale

- formuler des stratégies de prestation de services orientées vers les utilisatrices et acceptables pour certains groupes de population

- améliorer l'exécution des programmes pour qu'ils soient plus efficaces et plus viables sur le plan financier

- renforcer la capacité des directeurs des services de planification familiale d'utiliser la recherche opérationnelle pour identifier et résoudre les problèmes qui se posent au niveau de la prestation des services.

\section{Population Council}

Le Population Council, organisation non-gouvernementale à but non lucratif créée en 1952, réalise des programmes de recherche dont l'objectif vise à améliorer la santé en matière de reproduction et le bien-être des générations présentes et futures, ainsi qu'à réaliser un équilibre humain, équitable, et durable entre les populations et les ressources disponibles. Le Council, dont le siège se trouve à New York, analyse les questions et les tendances démographiques, et mène des recherches biomédicales afin de développer de nouvelles techniques contraceptives. Il travaille en collaboration avec les agences publiques et privées en vue d'améliorer la qualité des services de planification familiale et de santé en matière de reproduction et de promouvoir leur vulgarisation. L'Organisation apporte une assistance technique aux gouvernements afin de les aider à exercer une influence sur les comportements démographiques, communique les résultats des recherches menées dans le domaine de la population aux groupes concernés, et aide à la mise en place de structures de recherches dans les pays en développement. Le Council, qui est dirigé par un conseil d'administration dont les membres sont originaires de divers pays, apporte un soutien à tout un réseau de bureaux nationaux et régionaux.

Ce séminaire national a été realisé dans le cadre du deuxième Projet de Recherche Opérationnelle et d'Assistance Technique du Population Council. Le Projet de Recherche Opérationnelle est financé par "Agency for International Development" des Etats Unis, Bureau de la population, Contrat No. CCP-3030-C-00-3008-00, Stratégies destinées à améliorer la prestation des services de planification familiale. 


\section{SOMMAIRE}

Résumé en anglais

I. Introduction

II. Déroulement de l'atelier

2.1. Communications sur la situation actuelle de l'excision

2.2. L'experience des associations et ONG

2.3 Les religions monothéistes et l'excision

III. Synthèse des travaux

IV. Conclusion

V. L'avant-projet du plan national d'éradication de l'excision d'ici à l'An 2000

5.1 Les objectifs

5.2 Les stratégies de prévention et d'intervention

5.3 Les activités

VI. Budget

V1I. Annexe 


\section{EXECUTIVE SUMMARY}

The "Commissariat à la Promotion des Femmes" (CPF) is a Government entity under the authority of the Prime Minister which is mandated to address and promote the rights and interests of women in Mali. The CPF's commitment to advocate against the traditional practice of female circumcision, or female genital mutilation (FGM), was confirmed in 1996 by the creation of a National Committee of Action (CNA) for the eradication of harmful practices the well-being of women and children. The Population Council is an international nongovernmental organization whose mission is to seek to help improve the well-being and reproductive health of current and future generations around the world. The Population Council implements the Africa Operations Research and Technical Assistance (OR/TA) Project II which has the objective of improving reproductive health and health services in Africa through research and technical assistance.

The CPF, in collaboration with the Africa OR/TA Project II, organized a three-day national workshop on FGM which was held June 17-19, 1997 in Bamako. The objectives of the workshop were to present the results of a review of the literature on FGM and the actions taken to counter the practice in Mali; to present an update on the activities of six national NGOs working on issues surrounding FGM; and to develop and launch the CNA's Plan of Action for activities aimed at reducing the practice. In opening the workshop, the CPF pledged to advocate for the sexual and reproductive health of women by working alongside parliamentarians and non-governmental organizations, and stressed that the widespread violation of women's bodily integrity, especially through FGM, was a compelling cause for immediate and bold action.

The workshop brought together 85 participants, including eminent policy and decision-makers such as the Minister of Health, Solidarity, and the Elderly, the Minister of Justice, the Minister of Education, Representatives from the CPFand CAN, the Christian and Islamic religions, delegates from all regions of the countrym NGO, the Peace Corpsm donor agencies including USAID and UNICEF, the media, and multi-disciplinary experts in the field of reproductive health. During the workshop, participants agreed to undertake collaborative efforts among themselves to counter FGM because of its serious health consequences.

Some participants went further, urging the Government to ensure that sexual and reproductive rights form part of the legal and policy frameworks within the country. Other recommendations 
included raising awareness of FGM among the wider community, and developing an agenda for operations research.

The Government's commitment to reduce the practice of FGM was expressed through statements by the two high-ranking Government officials who chaired the opening and closing ceremonies. The Head of the CPF pointed out that "the Government could not eliminate hundreds of years of prejudice and discrimination against Malian women overnight. However, it surely can help them to stop this harmful traditional practice; but only by means of an interactive approach that takes into account the country’s social and cultural patterns."

The Minister of Health, Solidarity, and the Elderly, who chaired the opening and closing ceremonies told the gathering that "of all the harmful practices impacting on women's and children's health, FGM is the most widespread in rural as well as urban areas." He was referring to the results of the Mali 1995-1996 DHS which indicate a prevalence of $94 \%$ nationally among women of reproductive age. "The challenge is right there," he said, "to overcome these constraints, we must come up with a sustainable strategy for the total eradication of FGM in the country."

The workshop centered around the following themes: What do we know about FGM in Mali, and what strategies and national plan of action must we adopt to counter FGM? During the presentation of the literature review, the Population Council's national fellow outlined the characteristics and variations in FGM across regions and among ethnic groups - stressing that the regions of Kayes, Koulikoro, Sikasso, Segou, and the district of Bamako have undoubtedly the highest prevalence rates, whereas FGM is practically non-existent in the northern regions of Tombouctou and Gao. She expressed the hope that the political will and the constellation of various NGOs would be the national conscience of sexual and reproductive health. A document analyzing the results from the FGM module of the Mali DHS, which was prepared by the Population Council Office in Bamako, was presented by the Africa OR/TA Project II's Deputy Director.

The workshop led to the finalization of a national Plan of Action to the Year 2000 with the ultimate aim of eradicating FGM. Activities to be implemented under the national workplan will be coordinated by the CNA, and will include IEC interventions, research activities, advocacy, 
and training of specialists on FGM issues. The open, officially-supported criticism of FGM and the commitment expressed by renowned male opinion leaders to stop its practice were noteworthy features of the workshop and attracted substantial media coverage. 


\section{INTRODUCTION}

Les 17, 18, et 19 juin 1997, se sont tenues au Palais des Congrès de Bamako, les assises du Séminaire national pour la définition de stratégies d'éradication des pratiques néfastes à la santé de la femme et de l'enfant.

Organisé par le Comité National de Lutte contre les Pratiques Néfastes, cet atelier placé sous l'égide du Commissariat à la Promotion des Femmes, a bénéficié de l'appui technique et financier du Population Council.

L'atelier avait les objectifs suivants:

1. Renforcer la base de connaissance des participants sur la pratique de l'excision;

2. Analyser les différentes expériences sur le terrain afin de déterminer les réussites et les échecs de la lutte contre l'excision au Mali; et

3. Elaborer les éléments d'une stratégie nationale d'action qui servirait de base à l'élaboration d'un plan d'action pour l'éradication totale de l'excision, fondé sur une approche réellement participative et qui tient compte des réalités socioculturelles du Mali.

Les cérémonies d'ouverture et de clôture du séminaire étaient placées sous la présidence du Ministre de la Santé, des Personnes Agées et de la Solidarité. Elles se sont déroulées en présence de plusieurs personnalités à savoir:

- Commissaire à la Promotion des Femmes

- Commissaire à la Promotion des Jeunes

- Représentante de l'Organisation Mondiale de la Santé (O.M.S )

- Présidents des Hautes Institutions de la République

- Représentants des Missions Diplomatiques, Consulaires, des Organisations Internationales

- Délégation des bureaux du Population Council à Bamako, à Dakar, à Ouagadougou, et à New York. La liste des participants est jointe en annexe.

La Commissaire à la Promotion des Femmes a précisé que la redéfinition des stratégies d'éradication de l'excision était une nécessité eu égard aux faibles résultats enregistrés par les actions déjà menées. Elle a également rappelé les objectifs du séminaire et insisté sur 
l'importance que le Commissariat à la Promotion des Femmes accorde aux résultats des travaux dudit séminaire.

Dans son discours d'ouverture, le Ministre de la Santé, de la Solidarité, et des Personnes Agées, après avoir rappelé l'ancienneté et l'importance de la pratique de l'excision au Mali, a réaffirmé l'engagement du Gouvernement pour son éradication, un engagement qui s'est déjà traduit par la création d'un Comité National pour l'Eradication des Pratiques Néfastes à la Santé de la Femme et de l'Enfant. Il a noté que la définition des stratégies pertinentes était un véritable défi et qu'elle doit nécessairement tenir compte des différentes perceptions ainsi que de tous les aspects de la pratique de l'excision au Mali. Il a donc invité les participants à des débats francs et sans passion pour la réussite de l'atelier.

\section{DEROULEMENT DE L'ATELIER}

L'atelier s'est déroulé sous forme d'exposés suivis de débats, de travaux de goupe et de plénières.

\subsection{Communications sur la situation actuelle de l'excision}

Trois communications ont permis de faire le point de la situation actuelle de l'excision au Mali.

Il s'agit de :

2.1.1. La revue de la littérature

2.1.2. Exposé et discussions de l'analyse des données de l'EDS

2.1.3. L'Excision et la santé de la reproduction

2.1.1. La revue de la littérature et des actions menées sur le terrain par Mme Assitan Diallo du Population Council

Dans son exposé, elle a expliqué que les statistiques disponibles ne donnent qu'une image partielle des MGF au Mali, et que seulement quelques rares rapports de séminaires et rapports d'activités donnent des informations sur les activités entreprises jusqu'à présent. Sur la base des quelques données disponibles, elle a rappelé les caractéristiques des MGF au Mali et a présenté l'historique de la lutte contre les MGF. C'est ainsi qu'il apparaît que plusieurs types de MGF sont pratiqués au Mali, en l'occurence la clitoridectomie, l'excision et l'infibulation, 
et ces différents types concerneraient environ 94\% des jeunes filles et des femmes de 15 à 49 ans, de presque tous les groupes socioculturels du Mali, qu'ils soient de religions monothéistes ou de croyance animiste. L'âge moyen au moment de l'intervention est de 6 ans avec un nette tendance à la baisse en milieu urbain où la pratique a lieu quelque fois avant l'âge d'un an.

Parmi les raisons les plus invoquées pour justifier la pratique, on note le souci de préservation de l'identité culturelle, la conviction de respecter une prescription de l'islam et la volonté de contrôler la sexualité de la femme. Cependant, le conformisme semble être la seule explication dans la plupart des cas. Traditionnellement, l'intervention était pratiquée sur des groupes de jeunes filles du même âge par des exciseuses et s'accompagnait de cérémonies rituelles et d'une période de réclusion au cours de laquelle s'effectuait l'initiation des excisées. Avec l'évolution actuelle, l'excision se pratique quelquefois en milieu médical par le personnel de santé généralement sur des fillettes en bas âge, et a donc perdu sa valeur initiatique d'antan. Les conséquences gynéco-obstétriques, biologiques, et psychosomatiques de l'excision sont aussi mieux connues et servent de base à la sensibilisation dans le cadre de la lutte contre la pratique.

La communication révèle que la lutte contre l'excision date de plus de 30 ans et a connu des formes diverses : séminaires, conférences, débats à la radio ou à la télévision. Parmi les groupes cibles d'action contre l'excision, on peut noter le corps médical, les exciseuses traditionnelles, le corps enseignant, des associations féminines, les leaders d'opinion et surtout les religieux. Parmi les moyens de lutte, on note les efforts en matière d'information, education et communication (IEC), le lobbying des associations et ONG féminines, et la reconversion des exciseuses. Mme Diallo a dégagé des perspectives d'action contre l'excision et recommande notamment:

- l'intensification de la recherche;

- la poursuite de la sensibilisation;

- l'adoption d'un dispositif juridique;

- le secours aux personnes qui souffrent des conséquences de la pratique;

- la diversification du mouvement d'éradication par un élargissement à la base; et

- la diffusion de l'information scientifique et technique sur les MGF. 
Toutefois, certains intervenants ont émis des réserves sur quelques affirmations notamment celles relatives à l'existence de la pratique de l'excision en milieu Sonrhaï. L'auteur a précisé que l'affirmation ne concernait que Ansongo, dans la sixième région du Mali. Des discussions ont également longtemps porté sur le rôle des hommes, présentés tantôt comme totalement étrangers à la décision de l'excision, tantôt comme « demandeurs » de la pratique. Il a finalement été admis que l'attitude des hommes en tant que chefs de famille est déterminante dans la décision d'exciser ou non.

\subsubsection{Exposé et discussions de l'analyse des données de l'Enquête Démographique et de} Santé du Mali de 1996

Monsieur Diouratié Sanogo du Population Council de Dakar a présenté une analyse secondaire des données de la deuxième Enquête Démographique et de Santé du Mali (EDSMali II). Cette étude a été réalisée par M. Joël Tokindang et Mme Assitan Diallo du Bureau Population Council de Bamako.

Les sources de données permettant de mieux cerner le phénomène sont rares, donc les données de l'EDS/Mali II arrivent à point nommé et permettent de donner des statistiques assez fiables sur l'ampleur du phénomène ainsi que sur les variations et l'évolution à l'échelle du pays. Cette base de données permet d'avoir des informations sur les femmes, mais également sur leur filles aînées (pour les femmes qui en ont) par rapport à la pratique de l'excision. Ainsi, on a pu constater que la quasi totalité (94\%) des femmes âgées de 15 à 49 ans ont été excisées alors que bon nombre de leurs filles aînées ne l'ont pas encore été et ne le seront peut être pas. Le but de cette étude est de contribuer à une meilleure connaissance de la pratique, en allant au-delà des simples statistiques du moment afin de mieux appréhender les déterminants de l'excision au Mali. En fait, les méthodes d'analyse utilisées permettent de voir dans quelle mesure on peut croire ou non à un éventuel fléchissement des pratiques de MGF dans le pays.

Comme l'étude se veut prospective, elle s'est davantage focalisée sur l'excision des filles aînées car elles ne sont pas demandeuses d'excision -- bien souvent ce sont les parents qui les soumettent à ce rite. Ce constat soulève un certain nombre de questions:

- Quelles sont les caractéristiques qui semblent induire un penchant généralisé pour la pratique de l'excision au Mali? 
- Les filles aînées diffèrent-elles de leurs mamans quant aux risques qu'elles ont de connaître l'excision?

- Quelles sont les facteurs qui permettent de prédire l'évolution des risques de la pratique de l'excision au Mali, dans un avenir proche ou lointain?

On assiste d'une manière générale à un rajeunissement de l'âge d'excision au Mali. En effet, près de $70 \%$ des femmes âgées de moins de 30 ans sont excisées avant l'âge de 6 ans alors que cette proportion n'est que de 60\% chez les femmes les plus âgées de l'échantillon (45-49 ans). Pour revenir à certaines variables socio-économiques, on peut dire d'une manière générale que l'excision concerne moins les ethnies du Nord du pays à savoir les Sonraï et les Tamasheq. Par contre, c'est un phénomène qui touche la grande majorité des Malinké, des Peul, et des Bambara, ainsi que la quasi totalité des Soninké.

La variable niveau d'éducation ne permet pas de dégager des tendances nettes en faveur de la baisse du taux de prévalence des MGF. Un autre fait important à souligner, la ville (pour prendre l'exemple de Bamako) n'a vraisemblablement pas contribué à la disparition de la pratique. Au contraire, ce milieu assez cosmopolite est le théâtre des opérations les plus précoces comparativement à d'autres régions du pays.

Les analyses ont permis d'observer des différences assez significatives entre un certain nombre de variables et c'est notamment le cas de l'ethnie ou encore de l'éducation. Le facteur de prédiction qui semble le plus significatif par rapport à la pratique de l'excision est sans contexte celui du statut de la femme face à l'excision (être excisée ou non). Cette dernière caractéristique des femmes apparaît dans les analyses comme étant le facteur le plus déterminant et le plus discriminant: Les femmes excisées ont une propension 27 fois plus élevée de procéder à l'excision de leur fille aînée, que leurs consoeurs non-excisées. Cette situation laisse présager que la baisse du taux de prévalence de l'excision doit nécessairement passer par cette variable (endogène) ou plus exactement par une certaine rupture dans les chaînes de l'excision mère/fille. En fait, il ressort clairement que «l'excision entraîne l'excision ». 
Peut-on envisager une réelle baisse du taux de prévalence des MGF dans les années à venir?

$\mathrm{Au}$ vu des résultats des différentes analyses faites dans le cadre de cette étude, nous sommes tentés de répondre à la fois par oui et par non:

- Pourquoi oui? En effet, on constate que les femmes les moins âgées (donc des générations les plus jeunes) procèdent de moins en moins à l'excision de leur fille aînée.

- Pourquoi non? Au niveau des intentions, on a constaté une certaine homogénéité dans les opinions des femmes (tout âge confondu) pour la poursuite de la pratique de MGF. Les femmes favorables sont en proportion plus nombreuses que celles qui suggèrent l'arrêt de la pratique.

Si les intentions ne se limitent qu'au niveau des opinions, on peut toujours espérer une certaine baisse de prévalence du phénomène au Mali, mais il est difficile de déterminer dans quelle proportion, et dans combien de temps. Pour espérer arriver un jour à éradiquer le phénomène, il faudrait un grand effort de sensibilisation auprès de la population toute entière sur les dangers que courent les femmes soumises à cette épreuve. Comme il apparait plus que jamais que l'excision est un phénomène de société, les campagnes de lutte contre les MGF doivent non seulement être orientées vers les hommes et femmes analphabètes qui vivent en majorité dans le milieu rural, mais également vers les hommes et femmes instruits qu'on rencontre le plus souvent dans les centres urbains.

\subsubsection{L'excision et la santé de la reproduction}

Le thème sur l'excision et la santé de la reproduction a été exposé par deux gynécoobstétriciens: le Dr. Amadou Dolo, Gynécologue Obstétricien, et le Dr. Bouréima Maïga également Gynécologue.

Les conférenciers ont mis en évidence que l'excision était bien une pratique néfaste à la santé, avec des conséquences immédiates ou tardives. Parmi les conséquences immédiates, on note les hémorragies, les infections, la lésion des organes de voisinage, les douleurs; d'autres conséquences se révèlent souvent de façon tardive: les déformations vulvo-vaginales, les infections gyneco-urinaires, les douleurs lors des rapports sexuels, la frigidité, l'infécondité et la stérilité. En conclusion, ils ont recommandé de dénoncer l'excision et d'informer les populations sur ses conséquences, d'éduquer afin de reconvertir les mentalités avant l'adoption d'une loi. 
Les débats ont été particulièrement animés notamment sur le choix de la terminologie "mutilation" au lieu de "l'excision", la question de la responsabilité des exciseuses, les mesures à prendre contre la médicalisation et la nécessité d'adopter une loi. C'est ainsi que la terminologie "mutilations génitales féminines" (MGF) est apparue pour certains comme pouvant comporter une certaine connotation négative qui peut rendre son utilisation inefficace surtout lorsqu'elle sera traduite dans les langues nationales. D'autres participants ont estimé que seule cette terminologie recouvrait les différentes réalités de la pratique de l'excision.

\subsection{L'expérience des associations et $\mathrm{ONG}$}

\subsubsection{L'expérience de l'Association des Juristes Maliennes (AJM)}

Madame Ba Ami Boudiè Traoré du bureau de l'AJM a présenté l'expérience de son organisation dans la lutte contre les mutilations génitales féminines, une action qui s'inscrit dans le cadre des stratégies des droits de l'homme. L'AJM a été impliquée dans la lutte contre les mutilations génitales féminines à travers les actions de la clinique juridique qui est un centre d'information, de consultation et d'assistance juridique pour les femmes.

L'AJM a été saisie à plusieurs reprises par des femmes qui faisaient l'objet de procédure de divorce intentée contre elles par leur époux, ou qui étaient elles mêmes initiatrices de procédures contre leur mari suite à une situation d'abandon ou de répudiation par ces derniers. Lors des débats en chambre de conseil et sans violer le secret de l'instruction, les hommes assez souvent eurent comme argument que la femme était frigide ou refusait purement et simplement les rapports sexuels. Ils estimaient qu'ils ne pouvaient pas s'occuper d'une femme qui ne leur apporte aucune satisfaction sur le plan sexuel. Ces constats se sont multipliés par le canal de certaines femmes qui disaient éprouver des difficultés à entretenir des rapports sexuels réguliers avec leurs maris. Certains avocats ou juges membres de l'AJM ont également fait savoir qu'elles avaient l'habitude de rencontrer des femmes victimes de cette situation. Des présidents de juridiction ont souvent dirigé des femmes sur la clinique juridique en demandant à l'AJM de les aider à préserver leur foyer. A l'issue des entretiens

avec ces femmes, il est ressorti que toutes ces femmes ont été excisées et qu'elles ressentent encore des séquelles physiques ou/et psychologiques de la pratique. 
C'est ainsi que l'AJM a pu se rendre compte par elle-même que l'excision est susceptible d'engendrer pour les femmes qui l'ont subi, des conséquences graves sur leur vie. Une analyse plus approfondie a permis de comprendre qu'au-delà de la femme, les conséquences rejaillissent aussi sur l'époux qui est obligé de se remarier, et sur les enfants qui courent le risque de devenir des petits délinquants parce que privés d'une vie familiale normale suite à la séparation de leurs parents.

Lorsqu'elle a fait le constat que l'excision pouvait être source de problèmes pour les femmes, l'AJM a mené un certain nombre d'actions en vue de mieux appréhender et mieux cerner le problème:

a) Organisation d'une journée de réflexion sur le thème " L'excision et les droits de la femme "

Cette journée a regroupé des experts, des personnes ressources, des représentantes d'associations et d' ONG féminines, et des femmes victimes de la pratique de l'excision. A l'issue de la journée, le constat suivant a pu être fait:

- A la ville comme à la campagne, on assiste à la pratique de l'excision, et à sa persistance malgré la dénonciation en son encontre depuis un certain temps;

- $\quad$ Beaucoup de femmes présentent des séquelles liées à cette pratique;

- $\quad$ L'excision n'a aucun fondement religieux;

- $\quad$ Il n’y a jamais eu de poursuite ni de punition malgré des décès de bébés ou de fillettes suite à cette pratique.

b) Etude sur les aspects juridiques de l'excision par le Groupe d'Appui à la Réforme Juridique (GAREJ)

La principale question qui se pose est de savoir si l'excision ou toute autre forme de mutilation peut-être considérée à l'état actuel de la législation pénale malienne comme une infraction tombant sur le coup de la loi. Dans le cas contraire doit-elle faire l'objet d'une incrimination propre?

A l'analyse, il est vrai que l'excision comporte un acte matériel qui consiste à une atteinte à l'intégrité physique de la fillette. Il est difficile de déduire de là que tous les éléments constitutifs de l'infraction sont réunis notamment en disant qu'en pratiquant l'excision, 
l'exciseuse avait l'intention de nuire, de faire du mal à la victime. Cependant si l'intention de nuire fait défaut chez l'exciseuse, cette dernière par son manque d'habilité et de défaut de condition hygiénique souhaitable dans lesquelles elle pratique l'excision ou le fait de prodiguer à sa victime des soins non appropriés à la blessure constitue sur le plan pénal une faute, s'il s'en suit un fait dommageable se manifestant ici par les séquelles ou la mort. Dès lors, de l'avis de l'étude, rien n'empêche la victime ou les parents d'une victime de l'excision d'exercer des poursuites contre les auteurs et complices devant les tribunaux pour blessures involontaires en demandant réparation du préjudice. Mais dans ce cas ce n'est pas l'acte de l'excision qui est réprimé, mais le mauvais exercice et cela à la condition qu'il y ait faute, dommage et relation de cause à effet; alors que le but recherché par les adversaires de l'excision consiste à mettre fin à la pratique.

Les séquelles liées à la pratique de l'excision se manifestent très souvent bien des années après, or les actions en poursuite sont soumises à des délais qui, s'ils sont dépassés ne permettent aucune poursuite même si les autres conditions sont réunies. En ce qui concerne les instruments juridiques internationaux, ils n'ont aucune force juridique obligatoire sur les états signataires. Leur application au plan interne résulte de la volonté politique des signataires et du contexte sociologique.

S'agissant d'un phénomène de société les mesures préventives doivent se trouver dans un premier temps, au premier plan. Aussi les actions menées par l'AJM se résument dans les activités d'IEC. Cette action se fait en direction de toutes les couches de la population en particulier les femmes, les jeunes filles et les exciseuses.

\section{c) L'éducation des populations sur les droits de la personne humaine}

Il s'agit de faire comprendre aux populations que les droits de la femme sont une partie intégrante des droits de l'homme. Il s'agit également de leur faire comprendre que le droit à l'intégrité physique est un droit de l'homme et que la pratique de l'excision viole ce droit.

\section{d) Information des populations, surtout les parents et les exciseuses, sur les méfaits de l'excision}

Il est certain que si les mutilations génitales féminines persistent encore de nos jours, c'est que la majorité de la population ne connaît pas avec certitude les risques liés à leur pratique. 
En informant les populations sur les dangers de l'excision et les conséquences néfastes sur la santé de la mère et l'enfant, plus d'un parent réfléchirait par deux fois avant de livrer leur fillette aux mains des exciseuses. Il est également important de faire comprendre aux parents que l'excision de nos jours n'a aucune justification ni sanitaire, ni religieuse, sinon qu'à mettre en péril ou à briser la vie des femmes.

\section{e) Sensibilisation des parents mais surtout des exciseuses, qu'il est plus que grand temps de mettre fin à la pratique}

Blesser les personnes dans leur corps et leur vie ne peut pas être une profession digne d'exister. La sensibilisation va également à l'endroit des décideurs (députés, ministres, etc.) qu'il est plus que temps de réagir et d'adopter des mesures pouvant aider à mettre fin à l'excision. A cet effet, l'AJM a communiqué à tous les départements, services, et associations concernées, les résultats de son étude. Elle a proposé l'adoption pour l'avenir, des textes réprimant spécifiquement l'excision pour soutenir les actions d'IEC en cours.

\section{f) Les stratégies à adopter pour une meilleure prise en compte des droits de la femme dans une perspectives à long terme}

- Intensification et diversification des actions d'information, d'éducation et de sensibilisation. Ces actions doivent s'étendre aux religieux et aux leaders d'opinion. Elles doivent en outre concerner les élèves dans les établissements d'éducation de base. Il est primordial que la compréhension de ces activités soit accessible aux populations notamment en les tenant dans les langues nationales;

- Relecture de tous les textes de lois afin de les harmoniser avec les instruments juridiques internationaux;

- Mise en place d'équipes pluridisciplinaire afin de mener la lutte sur tous les aspects juridiques, sociologiques, sanitaires, et psychologiques;

- Proposition de loi réprimant l'excision pour appuyer la sensibilisation; et

- Prise en charge des femmes présentant des séquelles graves au niveau des centre de santé.

En conclusion, du point de vue de l'AJM, la sensibilisation de toutes les couches sociales et l'adoption d'une incrimination spécifique pour l'excision peuvent venir à bout des fausses croyances, des pressions sociales, et de tout autre facteur qui s'oppose à l'abandon de cette 
tradition. Toutes les contingences politiques et sociales actuelles recommandent à nos gouvernants de faire preuve de volonté politique plus affirmée dans la défense des droits de la femme. Cette volonté se traduit entre autres par:

- $\quad$ L'harmonisation entre les lois internes et de protection des droits de la femme;

- L'absorption par le droit positif interne de certaines pratiques coutumières néfastes à la santé de la femme.

En effet, il est certain que les campagnes de sensibilisation à elles seules ne sauraient faire abandonner ces pratiques à ceux ou celles dont elles constituent la principale source de revenus. Par ailleurs, les exciseuses doivent connaître les risques de poursuites juridiques applicables si elles sont incriminées en cas de complication. Elles ne se détourneront de ces pratiques qu'après avoir subi des sanctions répressives spécifiques à l'excision en tant qu'infraction. Le législateur ne doit plus se limiter à consigner par écrit dans une série d'articles, des voeux pieux ou des principes dont personne n'aurait à garantir l'application et le respect. Il faut qu'en plus, il soit institué un organe de surveillance qui sera chargé de veiller au respect et à l'application de la loi. Si maintenant, par conviction, une pratiquante suffisamment avertie de toutes les conséquences néfastes de l'acte décide de laisser tomber, elle serait la collaboratrice propice pour sensibiliser ses paires, surtout la jeune génération appelée à assurer la relève.

\subsubsection{L'expérience de l'Association Malienne pour le Suivi et l'Orientation des Pratiques}

\section{Traditionnelles (AMSOPT)}

Madame Sidibé Kadidia Aoudou, Présidente de l'AMSOPT a présenté l'expérience de l'AMSOPT en matière de conscientisation sur les mutilations génitales féminines. Après avoir présenté l'AMSOPT, ses zones d'interventions et ses objectifs, elle a fait le point des activités menées depuis 1995 et dégagé les perspectives pour l'avenir. L’AMSOPT est une ONG à but non lucratif créee en 1991 sous le récépissé nº72 du 31 mai 1991. L’AMSOPT n'a d'autres activités que celles relatives à la lutte contre les MGF.

La création de l'AMSOPT a été initiée à partir des événements de mars 1991 pour une continuation de l'action de l'ancien Comité National de Lutte Contre les Pratiques Traditionnelles Néfastes à la Femme et l'Enfant (le COMAPRAT). Le COMAPRAT etait un parapluie pour tous les intervenants sur le terrain (AMPPF, Croix rouge, Centre Djoliba, UNTM). L'AMSOPT intervient actuellement en1997 dans: 
- $\quad 40$ villages où intervient l'Organisation Plan International;

- $\quad 20$ villages de Sanankoroba;

- $\quad 10$ villages de Banamba;

- $\quad 10$ villages de Kangaba;

- $\quad 7$ cercles de la région de Kayes; et

- $\quad$ les centres de santé des Communes V et VI du District de Bamako.

Son objectif global est d'éradiquer les MGF d'ici l'an 2001. Ses objectifs spécifiques visent à:

- améliorer la santé de la reproduction;

- $\quad$ réduire le taux de mortalité infantile et de morbidité;

- $\quad$ faire prendre conscience des méfaits des MGF sur la santé de la reproduction à tous les groupes sociaux;

- $\quad$ amener les exciseuses à prendre conscience de leur responsabilité dans les cas d'hémorragie et de décès;

- $\quad$ amener les exciseuses à se reconvertir à d'autres activités génératrices de revenus;

- $\quad$ prendre en charge les victimes de l'excision; et

- $\quad$ mettre l'accent sur les pratiques traditionnelles positives.

Selon la présidente de l'AMSOPT, malgré les campagnes intensives d'information et d'éducation tant sur les plans nationaux qu'internationaux, les MGF persistent au Mali sous toutes les formes. C'est, consciente de cette persistance, que l'AMSOPT a dirigé ses recherches sur les raisons expliquant un tel comportement et qu'on peut résumer par ces constats:

- $\quad$ la MGF n'est pas perçue comme un problème de santé;

- les exciseuses ne se sentent pas responsables en cas d'hémorragie ou de décès (généralement attribué au mauvais sort);

- $\quad$ la décision d'abandonner l'excision est un problème familial ou communautaire;

- $\quad$ dans certaines localités du pays, le sang des excisées est utilisé pour les rituels ou comme adjuvant dans la préparation de certains poisons; et

- $\quad$ les raisons religieuses sont souvent évoquées pour justifier la pratique. 
Les activités menées par l'AMSOPT depuis 1995:

- $\quad$ Une prise de contact avec les leaders religieux et coutumier, avec les hommes, les jeunes femmes, les vieilles, les agents de santé avec une équipe multidisciplinaire;

- $\quad$ la formation des animateurs et superviseurs sur des techniques d'animation sur leur tâche et rôle; et

- $\quad$ la formation des personnes relais dans chaque village.

Des plans d'actions ont été élaborés à l'intention de ces différents intervenants. Toutes ces activités sont menées dans 40 villages où intervient l'organisation "Plan International". Trois ateliers d'information ont été organisés à l'intention des agents de santé et exciseuses dans les communes V et VI du District. Un autre atelier a été organisé à l'intention de 25 exciseuses. Ces activités ont donné déjà des résultats.

Trois mois après l'atelier d'information avec les exciseuses elles ont déposé les couteaux d'excision et ont crée une association dénommée "Ninka nanin ton ". Après réflexion, elles se sont mises d'accord pour mener des petites activités commerciales individuellement. Elles sont utilisées par AMSOPT pour des cas concrets de témoignages.

Sept jeunes filles ont été désinfibulées dans la clinique Mariam Diakité sur l'initiative de l'AMSOPT. Elles sont toujours sous traitement. Deux autres attendent d'être programmées pour consultation. Des femmes identifiées dans les villages de Sanankoroba souffrant de chélö̈des et de délabrement du périnée ont été consultées par le Gynécologue de l'AMPPF. L'AMSOPT souhaiterait construire un centre de formation pour la sous-région spécialisé dans le domaine des MGF. Ce centre aidera un jour l'ONG à s'auto-financer. En conclusion, la présidente de l'AMSOPT invite tous les intervenants à une franche collaboration pour qu'ensemble les objectifs puissent être atteints.

\subsubsection{L'expérience de l'Association pour le Progrès et la Défense des Droits des Femmes}

\section{Maliennes (APDF)}

Madame Fatoumata Siré Diakité, présidente de l'APDF a présenté l'expérience de son organisation dans la lutte contre les MGF, une lutte qui s'inscrit dans le cadre des priorités de l'association dans la lutte contre les pratiques traditionnelles rétrogrades et néfastes aux femmes et aux filles. Cette association considère les MGF comme la pire des violences à 
l'égard des femmes. Après avoir rappelé les différentes formes d'excision pratiquées au Mali, la présidente de l'APDF a fait le point des activités menées par son association depuis 1993, pour l'abolition de la pratique. C'est ainsi que l'APDF a organisé plusieurs sessions régionales décentralisées d'information, d'éducation et de sensibilisation des femmes exciseuses dans les régions de Kayes, Mopti, Koulikoro, Sikasso et dans le District de Bamako.

L'APDF a initié un programme pilote de reconversion socio-économique des exciseuses à Ségou. Ce projet financé par l'Ambassade des Etats Unis d'Amérique a porté sur:

1. l'identification par les femmes elles-mêmes d'activités génératrices de revenus à entreprendre à Ségou;

2. la formation des femmes exciseuses identifiées et retenues pour le projet à la maîtrise des techniques de mise en marche et de gestion d'une machine décortiqueuse; et

3. l'achat et l'installation des machines dans deux quartiers identifiés à Ségou.

Dix femmes exciseuses travaillent autour des deux machines implantées à Dar-Es-Salam et Pélégana.

En plus de ces activités économiques, neuf comités permanents d'information et de sensibilisation ont été installés et les exciseuses sont membres de ces comités permanents. Leur rôle est d'organiser des séances de causeries-débats dans les quartiers autour des MGF, leurs conséquences néfastes sur les droits et la santé de la femme et de la fille, à l'aide de témoignages des victimes et prestataires de services. Il faut signaler qu'il y a quelques jours seulement, des exciseuses de Mopti et de Bandiagara ont remis leurs couteaux à l'APDF, en gage de leur engagement à abandonner cette pratique.

L'APDF a toujours mis l'accent sur la pratique des MGF au cours de la Commémoration des seize jours de Campagne Internationale de Lutte Contre les Violences Faites aux Femmes (16 novembre - 10 décembre). Elle a organisé des conférence-débats dans les établissements secondaires sur les MGF, en relation avec les droits humains et les deux Conventions internationales (CEDAW - CRDE). Des articles ont été publiés dans la «Voie de la Femme », un magazine de l'APDF sur la question des MGF. L'APDF a identifié surtout les jeunes scolaires et les enseignantes/enseignants comme groupes cibles pour ses actions/activités d'information et de sensibilisation, afin d'aboutir à un changement progressif de 
comportement et de mentalité en faveur de l'abolition des MGF au Mali. C'est ainsi qu'elle a organisé le 8 mars 1997, un concours de meilleurs slogans et de meilleurs dessins sur «l'abolition de l'excision au Mali ». Des prix ont été décernés aux lauréat(e)s. Les slogans et dessins retenus serviront de supports visuels pour l'APDF dans ses activités contre les MGF. Une activité de recherche est en cours au niveau de l'APDF afin d'identifier les conséquences néfastes à long terme des MGF sur les femmes et les filles au Mali. L'APDF appartient à un réseau d'ONG qui se bat pour l'adoption de loi contre la pratique de l'excision au Mali, ceci viendrait compléter les actions et activités des ONG sur le terrain. Cette loi viserait en premier lieu le personnel du corps médical qui connaît les conséquences néfastes et continue à pratiquer les MGF pour des intérêts pécuniaires. Elle doit être aussi et surtout examinée sous l'angle juridique afin de combler le vide qui règne dans ce domaine. Le Code Pénal récent n'ayant prévu aucune disposition dans ce sens.

Les deux Conventions (CEDAW - CRDE) qui ont été ratifiées par le Mali, particulièrement celle relative aux droits de l'enfant, constituent des bases pour l'élaboration d'un texte juridique en faveur de l'abolition des MGF au Mali. En conclusion, l'APDF souhaite que les ONG travaillant sur le terrain et qui ont eu les résultats soient appuyées techniquement et financièrement afin de renforcer les acquis et en vue de l'obtention d'autres acquis à long terme. Pour cela, le Comité National d'Action devrait être une structure souple, ne se substituant pas aux ONG oeuvrant sur le terrain, mais plutôt un organe d'appui aux actions et activités de celles-ci. L'APDF est disposée à travailler avec toute organisation afin d'approfondir la réflexion sur cet aspect. Enfin l'APDF a en projet le montage de sketch en vidéo cassette sur les méfaits des MGF, sur les droits la santé des femmes et des filles au Mali. Elle voudrait aussi développer le matériel d'éducation et de sensibilisation sur les MGF, multiplier son programme au niveau de toutes les écoles au Mali, ainsi que le projet pilote de

Ségou. A moyen terme, l'APDF prévoit d'installer un téléphone « SOS » au siège de l'APDF, l'exemple ayant donné des résultats satisfaisants ailleurs.

\subsubsection{L'expérience de l'Association de Soutien au Développement des Activités de} Communication (ASDAP)

Madame Traoré Fatoumata Touré, Présidente de l'ASDAP a présenté l'expérience de l'ASDAP dans la lutte contre les MGF. Après avoir rappelé les différents types d'excisions pratiqués au Mali et les conséquences immédiates et à long terme sur la vie de la femme et de 
l'enfant, elle a indiqué l'approche utilisée par l'ASDAP, les points faibles de la stratégie utilisée, et la stratégie actuelle.

L'approche utilisée par l'ASDAP au départ, à travers ses projets en 1984/85, était d'introduire dans les curricula de formation des animateurs relais, des accoucheuses, et des matrones, le thème sur "les méfaits de l'excision". Les réactions étaient très timides car c'était un sujet tabou. Pour la persévérance dans la sensibilisation au niveau des villages, des causeries débats étaient animées sur le thème par les animateurs relais communautaires appuyés par le staff technique de l'ASDAP. Petit à petit les communautés ont commencé à être à l'aise avec le thème, avec l'appui des accoucheuses traditionnelles.

Les séances de recyclage annuel reprenaient le thème de l'excision à toutes les sessions. En 1989, sous l'égide de l'Union Nationale des Femmes du Mali, la présidente de l'ASDAP a animé toutes les conférences sur l'excision dans les sept cercles de la région de Koulikoro. L'approche au cours de cette grande campagne de sensibilisation était de sensibiliser tous les leaders communautaires (hommes et femmes), les agents socio-sanitaires, les administrateurs, la presse, les exciseuses et les accoucheuses traditionnelles sur les méfaits tant de l'excision que des autres pratiques néfastes (tabous nutritionnels, mariage précoce, etc.). Le point faible de cette stratégie a été l'interruption brutale du programme, ce qui a empêché d'apprécier à juste titre les effets réels des activités menées dans ce cadre. Cependant, l'intérêt que les populations ont porté au thème a encouragé l'ASDAP à réfléchir sur la continuation de la lutte contre cette pratique néfaste, dans ses programmes ultérieurs.

L'ASDAP, tenant compte des leçons tirées de son expérience de terrain, développe actuellement une autre stratégie. Cette stratégie est basée sur la sensibilisation progressive des communautés avec intégration des exciseuses dans les programmes d'IEC pour lutter contre l'excision. Afin d'y parvenir, diverses activités sont menées:

- L'organisation des journées de concertation avec les exciseuses traditionnelles, les agents de santé, les leaders communautaires (hommes et femmes), les différentes structures organisées dans les localités pour échange d'information sur l'excision et ses méfaits; 
- L'utilisation des exciseuses traditionnelles comme animatrices relais pour animer des séances d'IEC dans les communautés (causeries, visites à domicile...) après formation par le staff de l' ASDAP;

- La promotion de petits crédits pour les exciseuses reconverties afin de pallier au déficit causé par l'abandon de leur métier et l'utilisation en guise de motivation; et

- $\quad$ Le suivi constant.

\subsubsection{L'expérience du Comité d'Action pour les Droits de la Femme et de l'Enfant} (CADEF)

Madame Diallo Fatoumata Binta Diop a présenté le CADEF, une ONG créée en 1990, dont l'objectif est de diffuser la convention relative aux droits de l'enfant et la convention relative à l'élimination de toutes les formes de discrimination à l'égard des femmes. Elle a rappelé les actions du CADEF pour les droits de la femme en matière de santé et de reproduction. C'est dans ce cadre que le CADEF a mené de nombreuses activités d'IEC et de sensibilisation contre les MGF, et a aussi mené une série d'enquêtes à Kayes, Ségou, Sikasso et dans certaines communes du District de Bamako. En conclusion, le CADEF souhaite que l'atelier puisse dégager un cadre de référence pour les futures actions de lutte contre l'excision.

\subsubsection{L'expérience du Centre Djoliba}

Le Centre Djoliba a une longue expérience dans la lutte pour l'éradication de l'excision au Mali. L'action du Centre Djoliba a connu plusieurs phases. La première phase qu'on peut situer entre 1984 et 1986 a été consacrée à l'identification du matériel pédagogique, à l'information et à la sensibilisation à travers des actions d'animation de groupe, des conférences, et la formation de personnes relais dans les quartiers de Bamako et dans les villages environnants. De 1987 à 1989, des sessions d'animation ont été organisées dans les établissements scolaires et le Centre Djoliba a étendu son action sur l'ensemble des régions du Mali (à l'exception de celles du Nord). Pendant cette période des séminaires ont été organisés à l'intention des enseignants et de nombreuses causeries débats publiées et organisées au Centre. Mais l'action déterminante a été la soutenance de la thèse d'une étudiante en médecine sur les «Aspects sociaux sanitaires de l'excision au Mali » qui a permis une reconnaissance officielle et scientifique des caractères nocifs de l'excision sur la femme et l'enfant. 
Le Centre Djoliba poursuit ses activités depuis cette date en collaboration avec de nombreuses associations et ONG telles que l'APDF, l'AMSOPT, le CADEF, la COFESFA, et la clinique juridique de l'AJM. La méthode d'approche utilisée par le Centre Djoliba est une démarche participative et responsabilisante basée sur une pédagogie qui permet la transmission de savoir par le questionnement et l'image, pour amener progressivement la population à réfléchir sur sa propre situation et à trouver des solutions. Des résultats appréciables ont été obtenus grâce au Centre Djoliba dont les actions ont eu un impact réel sur les mentalités, même s'il y a eu souvent des aspects négatifs. En dépit des difficultés rencontrées, le Centre continue son action et a beaucoup de perspectives pour l'avenir.

\section{a) Résultat obtenus}

La multiplication des sessions d'information et d'éducation populaire ont favorisé une prise de conscience chez les populations et une ouverture des mentalités populaires et des responsables politiques et de la santé; ceci fait que le sujet n'est plus tabou.

- $\quad$ l'utilisation d'un matériel simple et adapté, permet de mettre hommes et femmes ensemble pour discuter le sujet;

- la formation d'animateurs et d'animatrices relais pour poursuivre la lutte et évaluer progressivement les changements internes dans les comportements;

- $\quad$ le Centre Djoliba est de plus en plus sollicité par des associations et organismes nationaux et internationaux pour un appui pédagogique et une formation en animation dans plusieurs domaines;

- $\quad$ l'abandon de la pratique par une exciseuse ayant bénéficié des animations du Centre Djoliba.

\section{b) Impact des actions}

Les intervenants se sont multipliés et dans des disciplines diverses. Le Centre Djoliba est devenu membre du Comité Inter-Africain de Lutte contre les Mutilations :

- $\quad$ la réduction légère de la pratique peut être constatée;

- le sujet n'est plus tabou, l'excision est abordée avec toutes les couches de la population, le problème est mieux connu au Mali;

- $\quad$ la pratique a perdu son caractère mystique;

- $\quad$ la majorité rejette l'excision mais n'a pas encore le courage de l'arrêter;

- $\quad$ les non excisées ne se sentent plus indexées. Certaines se sentent plutôt valorisées; 
- $\quad$ la constitution d'une banque de données; et

- $\quad$ les exciseuses se plaignent de la diminution de leur clientèle.

Ces actions ont eu aussi des effets non désirables :

- certaines femmes excisées se considèrent comme victimes, d'autres sont gênées par l'évocation sur la scène publique des problèmes intimes;

- $\quad$ un grand nombre d'intervenants agit sans toujours être bien documentés sur le sujet; des acteurs favorables aux MGF se sont appropriés des résultats de la lutte; comme par exemple, les agents de santé qui veulent institutionnaliser la pratique dans leurs tâches tout en prévenant ses conséquences infectieuses et certains marabouts préconisant la «Sunna » (la clitoridectomie) pour minimiser les dégâts de l'acte.

- $\quad$ les acquis de la lutte ont permis également de mettre le doigt sur des drames intimes (couples discordants, célibataires infibulées, déformations génitales).

\section{c) Difficultés rencontrées}

- $\quad$ difficulté de mise en application des acquis de la formation due à l'influence des vieilles personnes et plus particulièrement les belles-mères;

- $\quad$ lenteur dans les changements de mentalités;

- $\quad$ l'analphabétisme des femmes; et

- la pression religieuse constitue souvent un facteur de blocage quant à la mise en pratique de certains acquis.

\section{d) Perspectives}

- démultiplier et décentraliser les interventions à l'intérieur du pays;

- $\quad$ former des partenaires relais dans 4 régions du Mali;

- $\quad$ renforcer une campagne d'information publicitaire (tracts, affiches, radio);

- $\quad$ actualiser les recherches sur la pratique de l'excision selon les milieux socioculturels;

- $\quad$ impliquer plus de chefs religieux dans la lutte;

- $\quad$ organiser des séminaires régionaux et nationaux pour échanger les expériences avec les autres structures intervenantes;

- $\quad$ sensibiliser les exciseuses; et

- $\quad$ créer des activités rémunératrices pour les exciseuses. 
L'année 1996 peut être considérée comme un tournant décisif dans la recherche de stratégies pour harmoniser les approches de langages et créer l'esprit de réseau. Le programme du Centre Djoliba évolue de plus en plus vers le suivi appui conseil pour la formation, la recherche, le développement de nouvelles approches, des outils didactiques, des terminologies pour renforcer les acquis et améliorer la qualité des interventions. Des recherches sont aussi en cours dans ce Centre pour une approche globale et différentielle «genre» sur les différents groupes cibles et les alternatives d'approche pour un développement durable et plus équitable. Le Centre Djoliba souhaitait entreprendre les activités suivantes jusqu'à la fin de l'année 1997:

- $\quad$ réformuler le problème dans ses dimensions multiples;

- $\quad$ harmoniser les approches;

- $\quad$ renforcer la concertation et la collaboration entre les intervenants;

- $\quad$ se positionner comme centre de référence en matière de compétence en ressources humaines et

- contribuer au développement d'une éthique professionnelle en matière de lutte contre les MGF.

En conclusion on peut retenir que les résultats obtenus sont surtout la reconversion des mentalités et le changement d'attitudes. Selon la division promotion féminine du Centre Djoliba, cela démontre le caractère essentiellement culturel de la pratique et la question qui se pose est de savoir s'il faut continuer à garder une coutume lorsqu'il est établi qu'elle affecte la vie de l'individu.

\subsection{Les religions monothéistes et l'excision}

\subsubsection{Le point de vue de l'église protestante}

Selon Madame Diakité Pauline, lorsque l'Eglise Evangélique Protestante est rentrée au Mali, les premiers missionnaires ont rencontré des populations très attachées à des pratiques telle que l'excision, le plus souvent entourée de mystique ou de mystère et donc peu conciliables avec une vraie foi. C'est pourquoi l'église a très tôt lutté contre l'excision et si la pratique persiste toujours on peut attribuer cela au fait que l'attitude des femmes chrétiennes qui continuent à perpétrer cette pratique rejoint celle de la plupart des femmes qui sans bien comprendre suivent les autres par conformisme. Les responsables des femmes chrétiennes 
organisent souvent des conférences sur le thème de l'excision au cours desquelles des spécialistes informent des femmes et les sensibilisent pour les inciter à abandonner la pratique. Ces actions de sensibilisation se poursuivent au sein de l'association panafricaine des femme chrétiennes.

\subsubsection{Le point de vue de l'église catholique}

Pour Madame Diallo Jeanne Marie Diakité, aucun passage de la bible ne parle de l'excision, par contre elle parle de la circoncision dans l'Ancien Testament Genèse 17 Verset 10. Dieu s'adressant à Abraham dit «Quiconque est parmi vous de sexe masculin devra être circoncis ». Cette circoncision était le signe de l'alliance entre Dieu et l'homme. Jésus aussi n'a pas parlé de l'excision. Il a lutté pour le bien-être de l'homme, il est venu pour sauver l'homme corps et âme. L'excision est une mutilation, car elle diminue sexuellement la femme. C'est une opération moralement interdite, parce qu'elle prive la femme d'un organe essentiel qui l'empêche de jouir pleinement de son corps, d'accomplir ses obligations d'épouse. L'excision est une injustice car pendant l'acte sexuel, l'homme se donne en entier à la femme, mais lui ne reçoit qu'un corps incomplet. Elle peut traumatiser la femme qui, à cause des douleurs pendant les rapports, peut refuser son mari (1 Corinthiens 7 à 15). L'homme doit disposer du corps de sa femme vise versa. L'épouse doit toujours accepter son mari, Dieu veut le bien-être du couple. Pour terminer, l'excision n'est pas recommandée par le Bible. Etant des vrais croyants, nous devons tout faire pour l'éradication de cette pratique qui porte atteinte à l'intégrité de la femme.

\subsubsection{Le point de vue de l'Islam sur l'excision}

Selon Monsieur El Hadj Abdoulaye Camara, Secrétaire aux relations intérieures du Bureau Exécutif National de l'Association Malienne pour l'Unité et le Progrès de l'Islam (AMUPI), l'excision est une vieille coutume dont il est difficile de situer l'origine.

Cependant plusieurs hadiths du Prophète Mohamed (PSL) font référence à l'excision notamment trois :

- " "quand deux circoncis ont des rapports, la grande ablution est obligatoire »;

- _ _ il y a cinq choses qui sont naturelles chez l'homme parmi les cinq figure l'excision $»$;

- _ _ à Oum Attiya le Prophète aurait recommandé de couper un tout petit 
peu et pas tout, parce que c'est une beauté pour la femme et un plaisir pour le mari».

Selon l'Islam l'excision n'est pas une obligation et la non excision n'est pas un péché. Cependant l'AMUPI conseille de s'en tenir aux recommandations du prophète et de consulter les médecins et les agents de santé avant de procéder à l'excision. L'AMUPI reconnaît cependant que plusieurs maladies sexuellement transmissibles peuvent être évitées par l'abandon de la pratique de l'excision : le Sida et les maladies sexuellement transmissibles. 


\section{SYNTHESE DES TRAVAUX}

Les discussions en plénière ont été l'occasion de large débat sur certaines questions :

\subsection{L'adoption d'une loi interdisant l'excision ou érigeant l'excision en infraction} spécifique dans le Code Pénal a été longuement discuté.

Pour certains participants, les campagnes de sensibilisation à elles seules ne suffiraient pas à faire abandonner la pratique de l'excision et les actions d'IEC doivent être soutenues par l'adoption d'une loi. Cependant les points de vue sont différents sur le contenu de cette loi. Pour l'AJM, les sanctions qui seront prévues doivent s'appliquer aux exciseuses; pour l'APDF, ce serait plutôt le personnel médical qui devra subir les sanctions. De nombreux participants ont soulevé les difficultés d'application qu'une telle loi pourrait rencontrer dans la pratique si les mentalités ne sont pas suffisamment préparées. C'est ainsi que l'AJM propose pour surmonter ces difficultés, la mise en place d'un comité de suivi de l'application de la loi. Le Commissariat à la Promotion des Femmes a informé les participants de l'étude en cours sur l'opportunité d'une loi relative à l'excision; les résultats de cette recherche seront soumis au Comité National pour l'Eradication des Pratiques Néfastes à la Santé de la Femme et de l'Enfant.

\subsection{La question $d u$ choix de la terminologie mutilation génitale féminine dont} l'utilisation, surtout dans les langues pourrait soulever les problèmes de communication a été également discutée.

Certains participants ont estimé que seule l'utilisation de ce terme permet de prendre en compte les différentes réalités de la pratique. Pour d'autres au contraire cette terminologie comporte une connotation négative qui pourrait être mal comprise des populations qui pourraient y voir une critique de leur culture. Les différents points de vue exprimés n'ayant pas abouti à une conclusion définitive le séminaire a recommandé de consacrer une étude spécifique à cette question. La nécessité de poursuivre la recherche et d'approfondir les études réalisées sur un certain nombre de sujets a été reconnue par l'ensemble des participants; les domaines prioritaires de recherche ont pu être identifiées lors des travaux en commission. 
3.3 La constitution des réseaux a été également reconnue comme un aspect essentiel dans la stratégie d'éradication de l'excision.

C'est ainsi que plusieurs propositions ont été faites par les participants pour aider à la création de nouveaux réseaux, ou à l'intégration dans des réseaux existantant au niveau national, régional ou international.

Les structures suivantes ont été identifiées par les participants:

\section{Au Mali:}

* $\quad$ Réseau de lutte contre les MGF

* Comité National d'Action pour l'Eradication des Pratiques Néfastes à la Santé de la Femme et de l'Enfant

\section{Réseaux régionaux:}

* CI-AF : Comité Inter-africain pour l'Abolition des Pratiques Néfastes à la Santé des Femmes et des Enfants.

* RIAF/DLVF : Réseau Inter-africain des Femmes pour la Défense des Droits et la Lutte contre les Violences faites aux Femmes

* CAMS : Comité pour l'Abolition des Mutilations Sexuelles.

\section{Réseaux internationaux}

* RAINBO

* IPDF

* WILDAF

* COMADE 


\section{CONCLUSION}

Le but du séminaire était de contribuer à une reconnaissance de l'impact négatif de l'excision sur le bien-être physique, psychologique et social de la femme et de l'enfant au Mali. Il visait spécifiquement trois objectifs :

- $\quad$ renforcer la base de la connaissance des participants sur la pratique de l'excision;

- $\quad$ analyser les différentes expériences de terrain; et

- $\quad$ élaborer les éléments d'une stratégie nationale d'éradication de la pratique.

Les résultats obtenus dans ce sens sont positifs. La qualité des communications, des contributions personnelles et des échanges ont permis aux participants d'améliorer leurs connaissances sur différents aspects de la pratique de l'excision et de disposer de résultats de recherches sur l'excision.

La présentation des expériences des différents intervenants a permis aux participants de connaître les différentes activités menées sur le terrain, ainsi que les résultats obtenus et les difficultés rencontrées. La courte durée du séminaire n'a pas permis de disposer d'un plan d'action parfaitement établi; toutefois les domaines prioritaires de recherche et d'intervention ont été identifiés et un calendrier d'activités a été ébauché par les commissions de travail établies au cours de la rencontre.

Un groupe restreint de personnes ressources a finalisé l'Avant-Projet du plan national d'action, qui sera soumis pour appréciation auprès du Comité National d'Action pour l'Eradication des Pratiques Néfastes à la Santé de la Femme et de l'Enfant au Mali.

La section suivante du rapport présente cet Avant-Projet dans ses grandes lignes. 


\section{L'AVANT-PROJET DU PLAN NATIONAL D'ERADICATION DE L'EXCISION D'ICI A L'AN 2000}

\subsection{Les Objectifs}

\section{a) Objectif général}

Accélérer l'élimination de l'excision en vue d'améliorer la santé des femmes et des filles.

\section{b) Objectif spécifiques}

- $\quad$ réduire à zéro la proportion de filles et de femmes victimes de l'excision;

- $\quad$ apporter des changements positifs dans les attitudes, croyances, comportements et pratiques de ceux qui prennent les décisions dans la famille concernant l'excision;

- $\quad$ instituer des activités appropriées pour mobiliser des partenaires, en l'occurrence les dirigeants de communautés, groupes politiques, groupes de femmes et d'hommes, accoucheuses traditionnelles, agents de santé adolescents et les jeunes.

\subsection{Les stratégies de la prévention et de l'intervention}

Elle comprennent la sensibilisation, l'information, l'éducation et la communication (IEC), la recherche, la formation, la législation, l'encadrement thérapeutique et l'assistance juridique et judiciaire.

\subsection{Les activités sont réparties en cinq composantes}

\subsubsection{Elaboration des programmes}

- Procéder à une évaluation des activités de lutte contre l'excision. Il s'agit, au besoin d'une enquête, de faire le point des activités menées dans le domaine de l'excision, d'identifier les acteurs qui y interviennent et de faire une analyse des différentes méthodes d'intervention.

- Intégrer un module sur les MGF dans la formation des infirmiers et des sages-femmes, ainsi que dans les programmes d'éradication en matière de santé et dans des domaines connexes. Il sera procédé au recrutement d'un consultant pour concevoir des modules adaptés aux ordres d'enseignement, préparer les rencontres avec les responsables chargés de l'education pour la prise en compte de ces modules, et organiser des rencontres de sensibilisation. 
- Mettre au point des plans pour la mobilisation sociale, la sensibilisation et les activités de formation afin de fournir un appui technique aux ONG au niveau local pour ce qui est de l'intégration dans leurs activités des messages relatifs à la santé. Une équipe sera mise en place pour l'identification des besoins et l'élaboration de ces plans lors de la 1ère année du projet.

\subsubsection{Recherches}

- Rassembler les données épidémiologique de base, notamment sur la prévalence et l'incidence de l'excision, ainsi que ses conséquences. Vérifier l'utilité des informations obtenues et définir des cibles et des indicateurs de surveillance. Il s'agit là de mettre en place un système de collecte de données et de procéder à un recensement au niveau des services de santé, surtout des agents de santé villageois. Cette activité se déroulera tout au long du projet.

- Procéder à l'organisation d'études pour l'amélioration des connaissances sur le phénomène :

- $\quad$ étude des connaissances socio -culturelles des MGF lors de la 1ère année du projet;

- $\quad$ étude sur les connaissances attitudes et pratiques des communautés la 2e année du projet;

- $\quad$ étude sur les raisons de la pratique de l'excision au Mali la lère année du projet;

- $\quad$ étude sur la précellence de l'excision au Mali la 1ère année du projet;

- $\quad$ étude des stratégies d'approche à adopter au niveau communautaire la lère année du projet; et

- $\quad$ étude psychosociale des méfaits de l'excision sur les femmes la 2e année.

Ces études seront entreprises par des consultants.

Mettre au point un système de collecte de données sur les complications obstétriques et gynécologiques dues à l'excision. Il s'agit d'élaborer des supports de collecte, d'organiser des ateliers de sensibilisation du personnel sanitaire et de procéder à une enquête pour mieux cerner les méfaits de l'excision. Cette activité se déroulera tout au long de la vie du projet. 
Impliquer les communautés dans l'identification des problèmes physiques, psychologiques et émotionnels liés à l'excision. Il s'agira de procéder à l'organisation de missions d'information et de sensibilisation des communautés afin d'obtenir leur adhésion aux activités et les associer à l'identification des questions liées à l'excision. Ceci pour recueillir toutes les informations pouvant apporter une modification dans la perception et le comportement des populations face à ce problème. Cette activité a lieu les trois premières années du projet.

Collaborer avec les institutions sous-régionales et internationales engagées dans la recherche en matière de lutte contre les mutilations génitales féminines. Il s'agit d'organiser des missions d'étude, des échanges d'expériences, de participer aux ateliers et conférences sousrégionales et internationales sur le thème. Cette activité sera réalisée durant tout le projet.

Assurer la diffusion de l'information. Il s'agira d'organiser des émissions et des débats à la radio, mais également à la télévision, de produire des articles dans les journaux. Les radios régionales et locales seront mises à contribution. Cette activité aura lieu pendant toute la durée du projet.

Réalisation de Sketches. Il sera procédé à la réalisation de sketches pour renforcer les activités d'IEC et de plaidoyer en cours. Ces sketches passeront à la télé et feront l'objet de spectacle lors de certaines cérémonies telle que la journée mondiale de la femme; la journée du panafricanisme, etc. Cette activité sera menée en 1998, 1999, et en l'an 2000.

\subsubsection{Plaidoyer aux niveaux régional, national et communautaire}

- Apporter un appui aux initiatives visant l'élimination de l'excision menées par les ONG, les groupes de pression, les associations et clubs de femmes. Il s'agit d'appui matériel, financier et/ou logistique pour leur faciliter leurs activités de sensibilisation.

- Organiser des ateliers de sensibilisation pour les dispensaires de soins de santé et autres groupes afin de les former et de les encourager à plaider en faveur de l'élimination des mutilations sexuelles féminines. Des ateliers de sensibilisation seront aussi organisés à l'intention des groupements sociaux et des leaders des communautés, afin de les encourager à plaider en faveur de l'élimination de l'excision. 
- Créer des réseaux de groupes d'appui féminin. Des réseaux existent déjà. Il s'agit de renforcer ceux là et de contribuer à la création de nouveaux afin d'avoir des groupes de relais de sensibilisation et de favoriser l'échange d'information entre les intervenants en organisant des rencontres de réflexion. Cette activité commencera la deuxième année et se poursuivra jusqu'à la fin du projet.

- Faire appel au soutien des chefs traditionnels et autres dirigeants influents de la communauté. Organiser des rencontres d'information et d'échanges avec les groupements sociaux et les leaders d'opinion. Cette activité se fera à partir de la deuxième année du projet.

- Organiser des émissions radiophoniques et télévisées. Il s'agit de concevoir des documentaires et d'organiser des débats.

- Mettre en place des centres d'information, de conseil et d'assistance. Il est question ici de louer des locaux, de recruter du personnel pour animer les centres, apporter de l'assistance aux personnes, de former le personnel travaillant dans ces centres. Un centre sera créée dans chaque cercle de région.

- Organiser des activités de sensibilisation des décideurs (plaidoyer auprès des décideurs, gouvernements, parlementaires, leaders d'opinion). Il s'agit d'organiser des journées d'information, des missions de sensibilisation dans les régions et cercles, et des ateliers de sensibilisation des autorités et des responsables locaux. Un atelier sera organisé en 1997, deux en 1998.

\subsubsection{Education et formation des ressources humaines pour promouvoir le renforcemen des} capacités à tous les niveaux

- Organiser un séminaire annuel pour sensibiliser les cadres du Commissariat à la Promotion des Femmes. Apporter l'appui institutionnel et matériel aux organisations oeuvrant à l'élimination de l'excision. Il s'agit d'apporter l'appui technique à ces organisations lors de l'élaboration de leur programme d'activités et assurer la formation de leurs agents. 
- Appui au fonctionnement du Comité National d'Action pour l'Eradication des Pratiques Néfastes à la Femme et l'Enfant. Cet appui comportera l'achat de deux véhicules pour la supervision et le suivi de leurs activités, le paiement régulier de carburant, de fonctionnement et l'achat de fournitures et de mobiliers, le paiement de deux mobylettes et une provision financiere pour l'entretien des engins achetés.

\section{Information - Education - et Communication}

\subsubsection{Mise au point du matériel d'éducation et de formation}

- Concevoir des matériels didactiques et audio-visuels pour le support des campagnes de sensibilisation: affiches, tee shirts, brochures, boîte à images, dépliants etc...

- Adaptation du matériel et des programmes d'informations, utilisés actuellement, au contexte culturel Malien. Un consultant sera recruté pour ce travail.

- Recenser le matériel IEC existant au cours de la lère année du projet.

Le coût d'exécution du Plan d'Action est estimé à 389200000 F CFA. 


\section{BUDGET}

\begin{tabular}{|c|c|c|c|c|}
\hline \multirow[t]{2}{*}{ ACTIVITES } & \multicolumn{4}{|c|}{ ANNEES (milliers de F CFA) } \\
\hline & 1997 & 1998 & 1999 & 2000 \\
\hline Evaluation des activités menées & 3000 & - & - & - \\
\hline Intégrer module dans les programmes & - & 5.000 & 5.000 & - \\
\hline Mettre au point un plan de mobilisation sociale & 2000 & - & - & - \\
\hline Rassembler les données épidémiologiques & 3000 & 1.500 & 1.000 & 500 \\
\hline Organisation d'études de recherche & 12000 & 6.000 & - & - \\
\hline $\begin{array}{l}\text { Mettre en place un système de collecte des données } \\
\text { gynécologiques }\end{array}$ & 4000 & 2.000 & 2.000 & 1.000 \\
\hline Implication des Communautés & 5000 & 10.000 & 6.500 & 3.000 \\
\hline Collaboration avec les institutions sous régionales & 1500 & 3.500 & 3.500 & 2.000 \\
\hline Diffusion d'information & 6000 & 10.000 & 7.500 & 3.000 \\
\hline Réalisation de sketches & 2000 & 4.000 & 2.000 & 2.000 \\
\hline Appui aux initiatives visant à éliminer l'excision & 6000 & 8.000 & 8.000 & 4.000 \\
\hline Atelier de sensibilisation & 2500 & 5.000 & 5.000 & 2.000 \\
\hline Création réseaux groupes d'appui & 1000 & 3.000 & 2.000 & 1.000 \\
\hline Activités d'implication des chefs traditionnels & 1000 & 1.000 & 1.000 & 1.000 \\
\hline Organisation d'émissions radiophoniques & 1500 & 3.000 & 3.000 & 2.500 \\
\hline Mettre en place un centre d'information sur les MGF & 3000 & 15.000 & 15.000 & 15.000 \\
\hline Sensibilisation des décideurs nationaux & 1200 & 3.500 & 1.500 & 500 \\
\hline Ateliers de sensibilisation et formation des cadre du CPF & 2500 & 3.000 & 3.000 & 2.500 \\
\hline Appui institutionnel aux organisations concerneés & 5000 & 10.000 & 10.000 & 5.000 \\
\hline Appui au fonctionnement du Comité National d'Action & 2500 & 6.000 & 6.000 & 6.000 \\
\hline Concevoir le matériel d'information & 2500 & 10.000 & 10.000 & 10.000 \\
\hline Adapter le matériel éducatif & - & 3.500 & 2.000 & - \\
\hline Recenser le matériel d'IEC & 3000 & - & - & - \\
\hline Evaluation Finale du programme d'activités & - & - & - & 3.000 \\
\hline TOTAL & 118.200 & 115.000 & 94.000 & 94.000 \\
\hline COUT TOTAL: & & & .200 .00 & F CFA \\
\hline
\end{tabular}




\section{ANNEXE}

\section{Liste de Participants}

SE. Sophie SOW

Fatoumata ABBAS

Mme Ag Madina AHMED

Mme BAGAYOKO Mariam Bagayoko

MME BAH Aminata

Mme BA Aissata Kone

Mme BERTHE Nahan Kante

Chiaka BOIRE

Mme CAMARA Fata Maiga

CISSE Bréima

Mme CISSE Djeneba Maiga

Daouda CISSE

Aiche COULIBALY

Mme Assitan COULIBALY

Mme Mariam COULIBALY

MLLE Adriana DANIS

Mme Diallo Mama DIAKITE

Dr. Mariam DIAKITE

Marie DIAKITE

Assitan DIALLO

Mme DIALLO Jeanne

Pr Ali Nouhoum DIALLO

Mme DIARRA Aminata Diarra

Mme Djeneba DIARRA

DIARRA Kadiatou Samoura

Fatoumata DIOP

Mme Mariam DOUMBIA

Seydou DOUMBIA

Kate FLEMING

Mme FOFANA Ramata Sissoko
Amb. Burkina

CAFO Gao

CAFO Kidal

AEFEK

AJM

Commisariat à la Promotion des Femmes

MDRE DNMR

Radio KLEDU

Commisariat à la Promotion des Femmes

Commissariat à la Promotion des Femmes

CAFO Mopti

$\mathrm{CM} / \mathrm{MJ}$

ADAF Galle

Coordinatrice, CRPF Sikasso

Croix Rouge malienne

Volontaire Corps de la Paix

CAFO Ségou

Gynéco-obstétricienne, Clinique Mariam Diakité

\section{AFCM}

Population Council

AFCM

Médecin

Secrét. Relations extérieures CAFO Koulikoro

CRM Croix Rouge

Coord Rég Ségou

CADEF

MLI 95/PO6

Pharmacien, Population Council

UNICEF

Secrét. APDF Adminis. Coor. Femmes 
Mlle GABBY

Nick GOUEDE

Mme HAIDARA A Sangho

Amy HAMELIN

Merle HANAN

Inoussa KABORE

Mme KALOGA Djeneba Sow

Mme KEITA Fatoumata Doumbia

KEITA Mariam Toure

Sam KLEIN

Djeneba KONE

Mme KONE Virginie Moukoro

LY Aminata Mama Traore

Lamine MAHAMANE

Mme MARIKO Minata Sidibe

Susan MCLUCAS

Geeta NARAYAN

Mme Coulibaly Djeneba OUATTARA

Dr OUATTARA Aichata Diakite

Dr Diarra Aichata SAKO

SANGARE Oumou Fofana

Diouratié SANOGO

N' Deye M'Batio SENE

SIDIBE Kadidia Aoudou

Mme Aissa SOW

Madani A. TALL

Mme THIAM Foufa Diallo

Mme TIMBO Koudedia Sidibe

Mme Coumba TRAORE

TRAORE Fatoumata Touré

Mariam TRAORE

Mme TOURE Djeneba Camara

Mme TOURE Kani Sangare
Volontaire Corps de la Paix

Spécialiste en Communic. PCouncil New York

Coord. Rég. Gao

Volontaire Corps de la Paix

(I)NTACT

Médecin, Population Council

Ordre des Sage-Femmes

Prof de Biologie

SEKA BAARA TON Sikasso

Volontaire Corp de la Paix

CADEF

Centre Djoliba

$\mathrm{CM} /$ Commissariat à la Promotion des Jeunes

Remplace la cood. De Tombouctou CP-CRPF

Remplace la coord. De Koulikoro (APDF)

Centre Djoliba

UNICEF

CNRST

APDF/CAFO

Assistante du Prog. Sante Plan International Mali

PNLS

Démographe, Population Council

CAFO

AMSOPT

UNICEF

Radio GUINTAN

MESSRS/ISFRA

AMSOPT

INRSP

ASDAP

AMPPF

AFEM

Commissariat à la Promotion des Femmes 
Yacouba TOURE

Gabbriella YATES
SECO ONG

Corps de la Paix 